\title{
Improving Cross-Protection against Influenza Virus Using Recombinant Vaccinia Vaccine Expressing NP and M2 Ectodomain Tandem Repeats
}

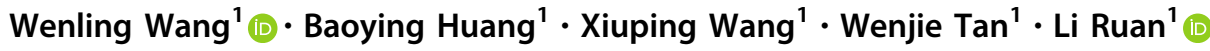

Received: 1 February 2019/Accepted: 29 April 2019/Published online: 25 June 2019

(c) Wuhan Institute of Virology, CAS 2019

\begin{abstract}
Conventional influenza vaccines need to be designed and manufactured yearly. However, they occasionally provide poor protection owing to antigenic mismatch. Hence, there is an urgent need to develop universal vaccines against influenza virus. Using nucleoprotein (NP) and extracellular domain of matrix protein $2(M 2 e)$ genes from the influenza A virus A/Beijing/30/95 (H3N2), we constructed four recombinant vaccinia virus-based influenza vaccines carrying NP fused with one or four copies of M2e genes in different orders. The recombinant vaccinia viruses were used to immunize BALB/C mice. Humoral and cellular responses were measured, and then the immunized mice were challenged with the influenza A virus A/Puerto Rico/8/34 (PR8). NP-specific humoral response was elicited in mice immunized with recombinant vaccinia viruses carrying full-length NP, while robust M2e-specific humoral response was elicited only in the mice immunized with recombinant vaccinia viruses carrying multiple copies of M2e. All recombinant viruses elicited NP- and M2e-specific cellular immune responses in mice. Only immunization with RVJ-4M2eNP induced remarkably higher levels of IL-2 and IL-10 cytokines specific to M2e. Furthermore, RVJ-4M2eNP immunization provided the highest cross-protection in mice challenged with $20 \mathrm{MLD}_{50}$ of PR8. Therefore, the cross-protection potentially correlates with both NP and M2e-specific humoral and cellular immune responses induced by RVJ-4M2eNP, which expresses a fusion antigen of full-length NP preceded by four M2e repeats. These results suggest that the rational fusion of NP and multiple M2e antigens is critical toward inducing protective immune responses, and the 4M2eNP fusion antigen may be employed to develop a universal influenza vaccine.
\end{abstract}

Keywords Influenza A virus (IAV) · Cross-protection · Recombinant vaccinia virus · Conserved antigen

\section{Introduction}

Seasonal influenza is an acute respiratory infectious disease that can cause serious health problems. Seasonal influenza epidemics caused by influenza $\mathrm{A}$ and $\mathrm{B}$ viruses result in 3-5 million severe cases and 290,000-650,000 deaths worldwide; $5 \%-10 \%$ of adults and $20 \%-30 \%$ of children

Electronic supplementary material The online version of this article (https://doi.org/10.1007/s12250-019-00138-9) contains supplementary material, which is available to authorized users.

\footnotetext{
Wenling Wang

wangwl@ivdc.chinacdc.cn

ruanlicdc@163.com

1 NHC Key Laboratory of Biosafety, National Institute for Viral Disease Control and Prevention, Chinese Center for Disease Control and Prevention, Beijing 102206, China
}

$\triangle$ Li Ruan suffer from flu annually (World Health Organization, WHO 2019). Seasonal influenza viruses undergo antigenic drift, which makes the conventional seasonal vaccines ineffective when the vaccine strains mismatch with the epidemic strains. Therefore, seasonal influenza vaccines must be updated regularly for effective prevention of influenza. The production time for conventional influenza vaccines can be lengthy. It takes 1-2 months to obtain vaccine strains using conventional reassortment technology or reverse genetics technology, followed by several more months for large-scale expansion of influenza vaccine using an ample supply of specific pathogen-free (SPF) chicken embryos. Generally, influenza vaccine production takes 3-6 months from the prediction of the epidemic strain to the ultimate production of the vaccine (Emanuel and Wertheimer 2006). For more effective prevention of influenza, new vaccines are warranted to induce crossprotection and long-lasting immune responses (Erbelding 
et al. 2018). Recently, great strides in influenza vaccine research have been made using conserved antigens of influenza virus. The highly conserved internal protein nucleoprotein (NP) and extracellular domain of matrix protein 2 (M2e) can induce cross-protection against influenza A virus, showing promise as candidate antigens for the development of a broad-spectrum influenza vaccine (Erbelding et al. 2018; Zhang et al. 2015; Kui et al. 2015). Previously, we expressed a fusion protein of NP and M2e (NM2e) in Escherichia coli and showed that immunization with NM2e formulated with aluminum hydroxide gel protected mice from a lethal challenge with heterologous influenza virus (Wang et al. 2012). Therefore, vaccination with recombinant NM2e fusion protein is a promising strategy for the development of a universal influenza vaccine. However, a new expression system is required to further optimize the NP- and M2e-based vaccine.

The vaccinia virus (Tiantan strain) was developed in China as a vaccination agent against smallpox, as documented by WHO (Fenner et al. 1988). This strain was used to inoculate several individuals for $\sim 50$ years, playing a key role in the eradication of smallpox in China (Ruan 2013). The Tiantan strain has favorable gene vector and vaccine characteristics, including a powerful multiplication capacity, wide host range, high capacity for insertion of a foreign gene, noncarcinogenicity, and induction of longlasting immunity. Using the $T K$ gene system of the Tiantan strain, this vaccinia virus has been developed successfully as a viral vector for various vaccine applications (Ruan 2013) including hepatitis B virus (Chuai et al. 2018), hepatitis C virus (Wen et al. 2013), and severe acute respiratory syndrome coronavirus vaccines (Yan et al. 2009). We have developed recombinant vaccinia viruses based on NP, M1, M2, and PB1 of influenza A virus (Huang et al. 2012; Wang et al. 2007, 2015). Several of our NP-based recombinant vaccinia viruses have induced a protective immune response in BALB/c mice. To develop a vaccine with broader protection, we constructed recombinant vaccinia viruses expressing various combinations of NP and M2e to exhibit their maximal antigenicity. BALB/c mice were immunized with the recombinant viruses to measure NP- and M2e-specific humoral and cellular immune responses as well as protective effect against lethal challenge with a heterologous influenza virus.

\section{Materials and Methods}

\section{Materials}

$\beta$-Gal (40-1a) monoclonal antibody (sc-65670) and NP monoclonal antibody (5D8) (sc-80481) were purchased from Santa Cruz Biotechnology (Santa Cruz, CA, USA).
M2 monoclonal antibody (14C2) (ab5416) and Alexa Fluor $^{\circledR}$ 488-conjugated goat anti-mouse polyclonal antibodies were from Abcam (Cambridge, MA, USA). BD Cytometric Bead Array (CBA) Mouse Th1/Th2/Th17 Cytokine kit was purchased from BD (Franklin Lakes, NJ, USA). Peptides NP55-69 (RLIQNSLTIERMVLS; H-2drestricted Th epitope), NP147-155 (TYQRTRALV; H-2drestricted CTL epitope), and M2e pooled peptides (peptides of residues 1-15, 5-19, and 9-23) were synthesized by Beijing SciLight Biotechnology Ltd. Co. (Beijing, China).

\section{Viruses}

Vaccinia virus (Tiantan strain) and influenza A virus A/Puerto Rico/8/34 (PR8) (H1N1) (Wang et al. 2015) were used in this study. Vaccinia virus (Tiantan strain) was amplified and titrated in primary chicken embryo fibroblasts (CEFs). The PR8 influenza virus was propagated in 9-day-old chicken embryos at $34^{\circ} \mathrm{C}$ for $48 \mathrm{~h}$, and the allantoic fluid was then collected and stored at $-70{ }^{\circ} \mathrm{C}$ until use. Mouse $50 \%$ lethal dose $\left(\mathrm{MLD}_{50}\right)$ titer of the influenza virus PR8 was assessed in BALB/c mice before conducting the challenge experiments.

\section{Construction of Plasmids and Recombinant Vaccinia Virus}

Plasmid pJSC1175 contains two DNA fragments (TKL and $T K R)$ derived from vaccinia virus Tiantan strain to facilitate homologous recombination with vaccinia virus in host cells. Between the two homology arms, there are p11 late promoter of vaccinia virus to regulate the lac $Z$ gene and p7.5 early/late promoter of vaccinia virus to regulate the target genes. The pJSC1175 was linearized at a BamH I restriction enzyme site immediately downstream the p7.5 promoter and further treated with calf intestinal alkaline phosphatase.

Plasmid pET30a-NM2e contains the fusion gene of fulllength $N P$ (498 amino acids) and the succeeding M2e (23 amino acids) from influenza A virus, A/Beijing/30/95 (H3N2) (BJ95) (Wang et al. 2012). The NP and M2e fusion gene (referred to as NPM2e) was amplified from plasmid pET30a-NM2e by polymerase chain reaction (PCR) to introduce $B a m \mathrm{H}$ I site at each end. The NPM2e was digested by $B a m \mathrm{H} \mathrm{I}$, and inserted into the linearized plasmid pJSC1175. The resulting plasmid carrying NPM2e at the same orientation as the 7.5 promoter was designated as pJSC1175-NPM2e.

From plasmid pJSC1175-NPM2e, $M 2 e$ and $N P$ genes were amplified respectively, and fused $(M 2 e$ preceding $N P)$ by PCR to generate a fusion gene, $M 2 e N P$. DNA fragment containing four copies of M2e (from BJ95) was amplified from plasmid, pET9a-H4M2e, and fused to the $5^{\prime}$ terminus 
of full-length $N P$ gene by PCR, resulting in fusion gene, $4 M 2 e N P$. The DNA fragment containing four copies of $M 2 e$ was also fused to the $5^{\prime}$ terminus of a $N P$ gene (from BJ95) truncated at both ends (referred to as NPs, encodes 314 amino acids) that was amplified from plasmid pET9aNPsM2e by PCR. The resulting fusion gene was named $4 M 2 e N P s$. As the fusion genes M2eNP, 4M2eNP, and $4 M 2 e N P s$ were engineered with $B a m \mathrm{H}$ I site at each end through PCR, they were cloned into linearized pJSC1175 as described above. The resulting plasmids were pJSC1175-M2eNP, pJSC1175-4M2eNP, and pJSC11754M2eNPs, respectively.

Recombinant vaccinia viruses were generated via homologous recombination, as described by Wang et al. (2015). Briefly, CEFs were infected with vaccinia virus Tiantan strain at a multiplicity of infection of 0.01-0.1, followed by transfection with recombinant pJSC1175 plasmids containing fusion genes of $N P$ and $M 2 e$. Subsequently, recombinant vaccinia viruses were screened by blue-white selection. Viral DNA was isolated from each recombinant vaccinia virus to confirm the presence of fusion genes (NPM2e, M2eNP, 4M2eNP, and 4M2eNPs) by PCR and sequencing of PCR products. The confirmed recombinant vaccinia viruses were produced on a large scale in CEFs. The control recombinant vaccinia virus RVJ1175 was generated using the same method with the empty pJSC1175 vector.

To confirm the expression of NP and M2e by the recombinant vaccinia viruses using indirect immunofluorescence, A549 cells grown in 48-well culture plates were infected with each strain of virus at 30-40 plaque-forming unit (PFU)/well. At $48 \mathrm{~h}$ post infection, the cells were fixed with $4 \%$ paraformaldehyde, and the expression of $\beta-\mathrm{Gal}$, $\mathrm{NP}$ and M2e was confirmed using mouse monoclonal antibody (MAb) against $\beta$-Gal (40-1a), mouse MAb against influenza A virus M2 (14C2), and mouse MAb against influenza A virus NP (5D8). The signals were then visualized using Alexa Fluor ${ }^{\circledR}$ 488-conjugated goat anti-mouse.

\section{Vaccination and Challenge}

SPF female BALB/c (5-6 weeks old) mice were purchased from Beijing Vital River Laboratory Animal Technology Co., Ltd. (Beijing, China). All mice were bred under SPF conditions at the Institute of Laboratory Animal Science, Chinese Academy of Medical Science and Peking Union Medical College, Beijing, China. Mice were randomly grouped (16 mice/group), inoculated intramuscularly with $1 \times 10^{7}$ PFU recombinant vaccinia viruses (RVJ1175, RVJ-NPM2e, RVJ-M2eNP, RVJ-4M2eNP, or RVJ$4 \mathrm{M} 2 \mathrm{eNPs}$ ) at week 0 and week 4 in the bilateral gastrocnemius without the use of reagents or equipment.
Ten days after the second immunization, three mice from each group were retro-orbital bled and sacrificed. The spleens were removed aseptically and ground through a 200-mesh sieve. Spleen mononuclear cells (SMNCs) were obtained as described by Wang et al. (2015). The cellular immune responses induced in mice were evaluated by enzyme-linked immunospot (ELISPOT) assay (Wang et al. 2012) and the BD CBA Mouse Th1/Th2/Th17 Cytokine kit according to the manufacturer's instructions. Peptides NP55-69, NP147-155, or M2e pooled peptides were used in the ELISPOT and CBA assays. The sera were separated by centrifugation, and serum IgG titers were determined.

Two weeks after the second immunization, mice were anesthetized using sodium pentobarbital $(10 \mathrm{mg} / \mathrm{mL})$ at a dose of $60 \mathrm{mg} / \mathrm{kg}$ of body weight. Subsequently, 10-12 mice in each group were challenged with 20 times of mouse $50 \%$ lethal dose $\left(M^{2} D_{50}\right)$ of PR8 intranasally. Weight loss and mortality were monitored daily for 3 weeks after the challenge. Mice that lost 30\% of their initial weight were euthanized and recorded as having died.

\section{Statistical Analysis}

Statistical analysis was performed using SPSS (ver. 17.0; SPSS Inc., Chicago, IL, USA) and Prism (ver. 5.0a; GraphPad Inc., La Jolla, CA, USA). Log conversion was performed for antibody titers. Differences in antibody titer, ELISPOT, and CBA results among groups were analyzed using one-way ANOVA. The paired $t$ test and log-rank test were used to analyze differences in weight loss curves and survival rate curves, respectively. Differences with $P$ values $<0.05$ were considered statistically significant.

\section{Results}

\section{Construction and Identification of the Recombinant Vaccinia Viruses}

To generate fusion antigens with the highest potency for inducing cross-protection, we designed a variety of fusion antigen configurations by adjusting the fusion order of NP and M2e, increasing the copy number of M2e (4M2e), and truncating the length of NPs. The $N P$ and $M 2 e$ gene fragments of influenza A virus A/Beijing/30/95 (H3N2) were joined to create $N P M 2 e, M 2 e N P, 4 M 2 e N P$, and $4 M 2 e N P s$ using PCR (Fig. 1). Each amplified fusion gene fragment was cloned into plasmid pJSC1175 under the regulation of p7.5 early/late promoter. The resulting plasmids pJSC1175-NPM2e, pJSC1175-M2eNP, pJSC11754M2eNP, and pJSC1175-4M2eNPs were confirmed by restriction endonuclease digestion and DNA sequencing 


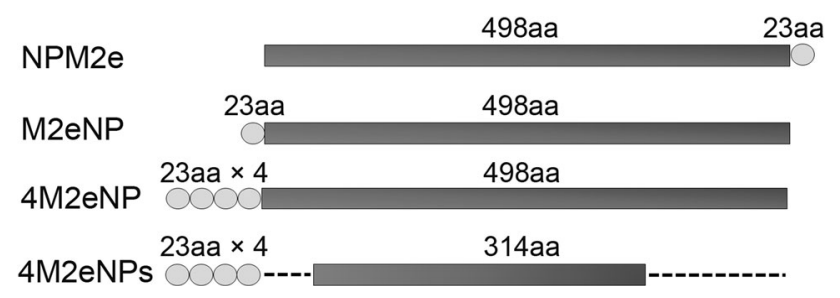

Fig. 1 Schematic diagram of NP and M2e fusions. NPM2e: the fulllength NP (498 amino acids) precedes one copy of M2e (23 amino acids); M2eNP: one copy of M2e precedes the full-length of NP; 4M2eNP: four copies of M2e precede the full-length of NP; 4M2eNPs: four copies of M2e precede truncated NPs (314 amino acids).

(data not shown). Each confirmed plasmid was transfected into CEFs infected by vaccinia virus Tiantan strain to facilitate homologous DNA recombination between plasmid and viral genomic DNA. Recombinant vaccinia viruses RVJ-NPM2e, RVJ-M2eNP, RVJ-4M2eNP, and RVJ4M2eNPs were screened by blue-white selection. The vaccinia virus DNA was extracted to amplify the target gene fragments using PCR. The inserted target genes were confirmed by sequencing of the PCR products.

\section{Immunofluorescence Identification of the Expressed Target Proteins}

A549 cells were infected with recombinant vaccinia viruses, and expression of the target proteins (NP and M2e) was identified by immunofluorescence. As a selective marker, $\beta$-galactosidase $(\beta-\mathrm{Gal})$ was expressed at similar levels in A549 cells infected by all recombinant vaccinia viruses. Except the control virus RVJ1175, all other recombinant vaccinia virus expressed M2e and NP in the infected cells. It seemed that RVJ-4M2eNPs showed lower expression of truncated NP than other recombinant vaccinia viruses carrying full-length $N P$ gene (Fig. 2). Flow cytometry analysis of the target protein expression showed similar results, and Western blot analysis confirmed target protein expression at the expected molecular weights (Supplementary Materials).

\section{Humoral and Cellular Immune Responses Induced by Recombinant Vaccinia Viruses}

To characterize the immunogenicity of fusion antigens expressed by the recombinant vaccinia viruses, five groups of $\mathrm{BALB} / \mathrm{c}$ mice were immunized with $10^{7}$ PFU of recombinant vaccinia virus at week 0 and 4 . Ten days after the second immunization, sera were taken to measure the antibody titer, and the spleens were removed aseptically to measure the cellular immune response. Mice immunized with the recombinant vaccinia virus RVJ-NPM2e and RVJ-
M2eNP showed strong antibody responses against NP, with lower titers of antibodies against M2e (Fig. 3A). RVJ4M2eNP induced strong antibody responses against both NP and M2e. While RVJ-4M2eNPs only induced a strong humoral immune response against M2e but not NP.

ELISPOT analysis (Fig. 3B) revealed that RVJ-NPM2e, RVJ-M2eNP, RVJ-4M2eNP, and RVJ-4M2eNPs induced more NP147-155-specific spot-forming cells (SFCs) than NP55-69-specific SFCs. RVJ-M2eNP induced significantly more NP147-155-specific SFCs than RVJ-NPM2e $(P<0.05)$ and RVJ-4M2eNPs $(P<0.01)$. RVJ-4M2eNP also induced significantly more NP147-155-specific SFCs than control virus, nevertheless there is no significant difference compared with RVJ-M2eNP. Except the control virus, all other recombinant vaccinia virus seemed to induce M2e-specific cellular immune responses (Fig. 3B). However, only M2e-specific SFCs induced by RVJM2eNP are significantly more than those induced by control virus $(P<0.01)$ and by RVJ-NPM2e $(P<0.05)$.

Cytokine measurements (Fig. 3C) showed that M2e pooled peptides stimulated SMNCs to secrete several cytokines including interleukin (IL)-2, IL-6, IL-10, and tumor necrosis factor. However, only immunization with RVJ-4M2eNP induced significantly higher levels of IL-2 and IL-10 cytokines than that with control (RVJ1175) when stimulation with the M2e pooled peptides.

\section{Protective Efficacy of the Recombinant Vaccinia Viruses}

We assessed the protective efficacy of the recombinant vaccinia viruses in the BALB/c mouse model. Two weeks after the second immunization, BALB/c mice were challenged with $20 \mathrm{MLD}_{50}$ of influenza A virus PR8, and then weight loss and survival rates were monitored for 3 weeks. The weight loss curve (Fig. 4A) showed that mice immunized with RVJ1175, RVJ-NPM2e, and RVJ-M2eNP experienced rapid weight loss, and all mice died by 9 days post-challenge. Mice immunized with RVJ-4M2eNPs also experienced rapid weight loss; several of these mice died soon after the PR8 challenge, while others experienced maximum weight loss by day 9 post-challenge followed by gradual recovery. Mice immunized with RVJ-4M2eNP experienced the lowest average weight loss (5\%) among the groups observed on day 6 post-challenge, followed by rapid recovery to the initial body weight at day 9 postchallenge. The survival rate results (Fig. 4B) showed that mice immunized with RVJ1175, RVJ-NPM2e, and RVJM2eNP died on days 7-8, 7-9, and 7-10, respectively, post-challenge. In contrast, $1 / 12$ mice in the RVJ-4M2eNP died on day 10 post-challenge, showing a final survival rate of $93 \%$. Mice immunized with RVJ-4M2eNPs died on days 7-11 post-challenge with a final survival rate of $30 \%$. 


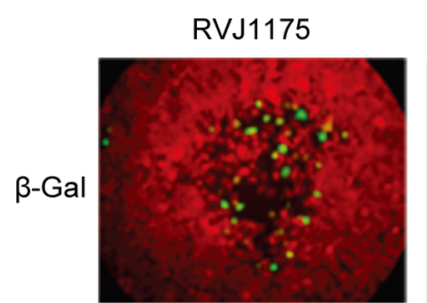

RVJ-NPM2e
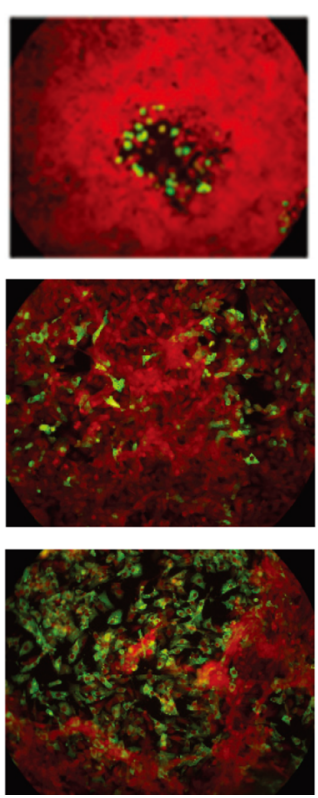

RVJ-M2eNP
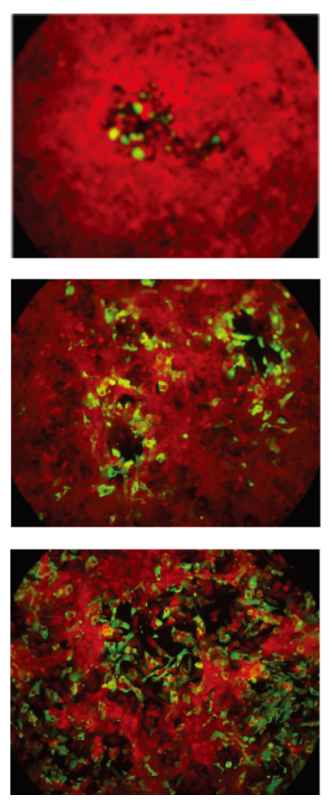

RVJ-4M2eNP
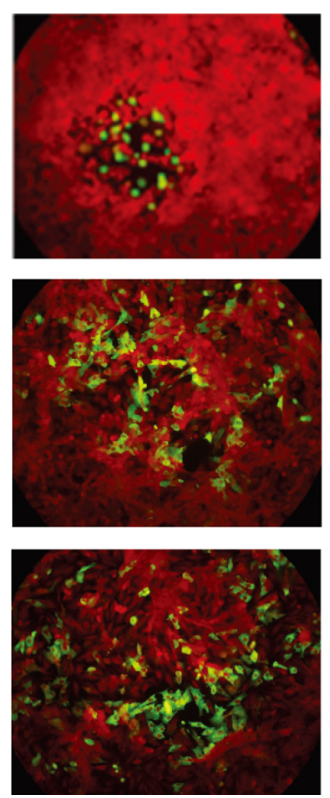

RVJ-4M2eNPs
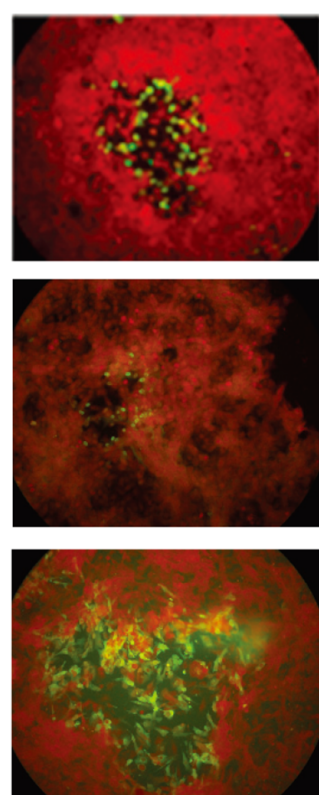

Fig. 2 Viral plaque formation and expression of NP and M2e fusions in A549 cells infected by the recombinant vaccinia viruses. A549 cells were infected by RVJ1175 (viral vector control), RVJ-NPM2e, RVJ-M2eNP, RVJ-4M2eNP, and RVJ-4M2eNPs. At $48 \mathrm{~h}$ post infection, the infected cells were immunostained by mouse

Statistical analysis showed that mice immunized with RVJ4M2eNP had significantly highest survival rate, and mice immunized with RVJ-4M2eNPs also had significantly higher survival rate than the remaining three groups (Fig. 4B).

\section{Discussion}

The extracellular domain of M2e is highly conserved among influenza A virus subtypes (Muñoz-Medina et al. 2015; Zebedee and Lamb 1988); thus, it was adopted as a target antigen in the development of a universal influenza vaccine. In the present study, a single M2e induced a poor M2e-specific humoral response (Fig. 3A, RVJ-NPM2e, RVJ-M2eNP). Four tandem repeats of M2e induced robust antibody response against M2e (Fig. 3A, RVJ-4M2eNP, RVJ-4M2eNPs), leading to a much stronger cross-protection than that of other groups against heterosubtypic H1N1 influenza virus challenge (Fig. 4B, RVJ-4M2eNP, RVJ$4 \mathrm{M} 2 \mathrm{eNPs}$ ). It had been proved that more robust M2especific immune response can be induced with multiple copies of M2e (Zhang et al. 2011; Zhao et al. 2010b; Zhou et al. 2012), and further enhanced by fusion with an appropriate protein carrier (Ma et al. 2013; Zhao et al. 2010a; Ebrahimi et al. 2012; Alvarez et al. 2013; Schotsaert et al. 2009). Our data suggest that M2e-specific monoclonal antibodies to detect $\beta$-galactosidase ( $\beta$-Gal) (upper panel), influenza NP (middle panel), and M2e (lower panel), followed by incubation with Alexa Fluor ${ }^{\circledR}$ 488-conjugated goat anti-mouse. Microscopic images were showed with 40-fold magnification.

antibody and cellular response played important roles in protection, and $4 \times \mathrm{M} 2 \mathrm{e}$ is more favorable than $1 \times \mathrm{M} 2 \mathrm{e}$ to induce protective immune response. M2e specific antibody may play a protective role by antibody-dependent cell-mediated cytotoxicity (ADCC), although it could not neutralize influenza virus (Kim et al. 2017; Lee et al. 2015). Besides, M2e specific cellular immune response may stimulate $\mathrm{CD} 4+\mathrm{T}$ cells to protect animals (Adlermoore et al. 2011).

NP has also been used as a target antigen to develop universal influenza vaccines (Huang et al. 2012; Li et al. 2013; Wang et al. 2014; Nahampun et al. 2015; Lei et al. 2015; Zheng et al. 2015). In the present study, a truncated NP protein lacking 50 amino acids at the $\mathrm{N}$ terminal and $\sim 70$ amino acids at the $\mathrm{C}$ terminal was designated as NPs. Although NPs retaining the middle part of NP with multiple conserved epitopes, it showed weaker immunogenicity compared with full-length NP (Fig. 3A, RVJ-4M2eNP, RVJ-4M2eNPs), and less protection in this experiment (Fig. 4B, RVJ-4M2eNP vs RVJ-4M2eNPs, $P<0.01$ ), probably because of less NP expression (Fig. 2, middle panel), suggesting that full-length NP is critical toward inducing protective immunity at least in BALB/c mouse. Previous research proved that human MAb against influenza NP played an important role in fighting low-dose infection of influenza virus (Fujimoto et al. 2016), and NP might exert a protective effect through ADCC (Kui et al. 
A

\begin{tabular}{|c|c|c|c|c|}
\hline NP & RVJ-NPM2e & RVJ-M2eNP & RVJ-4M2eNP & RVJ-4M2eNPs \\
\hline RVJ1175 & 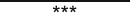 & 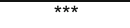 & 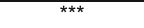 & ns \\
\hline RVJ-NPM2e & & $\mathrm{ns}$ & ** & $\star \star \star$ \\
\hline RVJ-M2eNP & & & * & $\star * *$ \\
\hline RVJ-4M2eNP & & & & *** \\
\hline M2e & RVJ-NPM2e & RVJ-M2eNP & RVJ-4M2eNP & RVJ-4M2eNPs \\
\hline RVJ1175 & $\mathrm{ns}$ & ns & $* * *$ & $* * *$ \\
\hline RVJ-NPM2e & & ns & $* * *$ & $* * *$ \\
\hline RVJ-M2eNP & & & $* * *$ & $* * *$ \\
\hline RVJ-4M2eNP & & & & ns \\
\hline
\end{tabular}

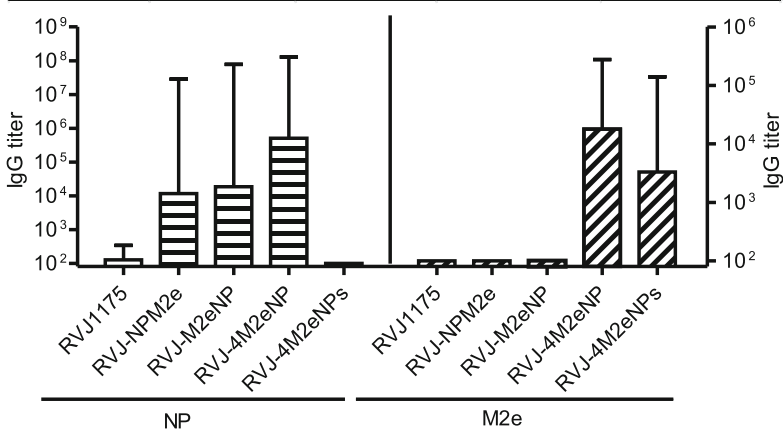

B

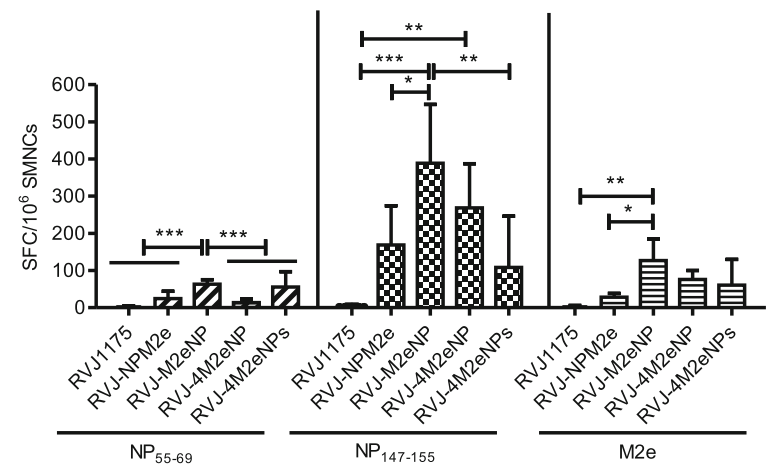

C

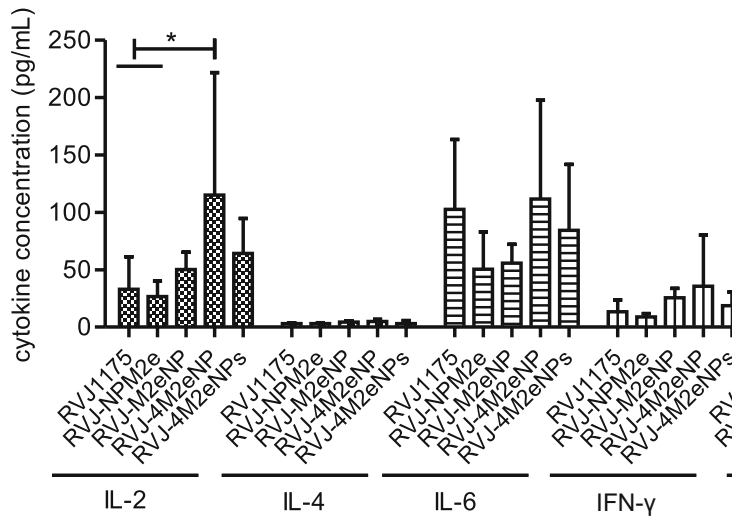

$1 \mathrm{~L}-2$

IFN-y
Fig. 3 Humoral and cellular immune responses in BALB/c mice immunized with recombinant vaccinia viruses. Mice were immunized intramuscularly with $10^{7}$ plaque-forming units of recombinant vaccinia viruses at weeks 0 and 4 . Group 1 (G1) mice immunized with RVJ1175 were served as negative controls. A Serum was obtained from three mice at 10 days post the second immunization and analyzed by enzyme-linked immunoassay (ELISA) for the presence of IgG antibodies specific for NP (left) or M2e (right), as described in "Materials and methods". The columns show geometric mean antibody titers, and the bars indicate the $95 \%$ confidence interval in each group ( $\mathrm{n}=3$ mice per group). Comparative results between two groups are shown in the upper table ( $n s$ not significant, ${ }^{*} P<0.05 ; * * P<0.01$; $* * * P<0.001$ by one-way ANOVA). B, C Cellular immune response in mice immunized with recombinant

2015; Jegaskanda et al. 2017). Moreover, NP contains numerous conserved CD4+ and CD8+ T-cell epitopes (MacLeod et al. 2013; Roti et al. 2008), which induce cross-T-cell immune and cross-protective responses (Hayward et al. 2015). The present study showed that NPspecific antibody and cellular response played important roles in protection. NP-specific antibody could not vaccinia viruses. Cytokines secreted from stimulated spleen mononuclear cells (SMNCs) were measured by enzyme-linked immunospot (ELISPOT) assay (B) and mouse Th1/Th2/Th17 CBA kit (C). All mice in each treatment group were sacrificed at 10 days post the second immunization. SMNCs were separated from mouse spleen samples, and $5 \mu \mathrm{g} / \mathrm{mL}$ NP55-69 (left B), NP147-155 (middle B), and M2e pooled peptides (right $\mathbf{B}$, and $\mathbf{C}$ ) were used as stimulants in the ELISPOT and CBA assays. After stimulation for $24 \mathrm{~h}$, the numbers of SMNCs producing interferon (IFN)- $\gamma$ (B) are presented as spotforming cells (SFCs) $/ 10^{6}$ SMNCs. The columns show the average SFCs $/ 10^{6}$ SMNCs, and the bars indicate the standard deviation of each group. Lines above two or more groups indicate that there was no significant difference. $* P<0.05$; $* * P<0.01$; $* * * P<0.001$ by one-way ANOVA.

neutralize influenza virus just as M2e. However, it may protect animals against challenge by ADCC, antibody-dependent cellular phagocytosis, or antibody-dependent complement deposition, and NP cellular immune response may stimulate $\mathrm{CD} 4+$ and $\mathrm{CD} 8+\mathrm{T}$ cells for protection (Guo et al. 2010; LaMere et al. 2011); the related mechanism is worthy of further investigation. 
A

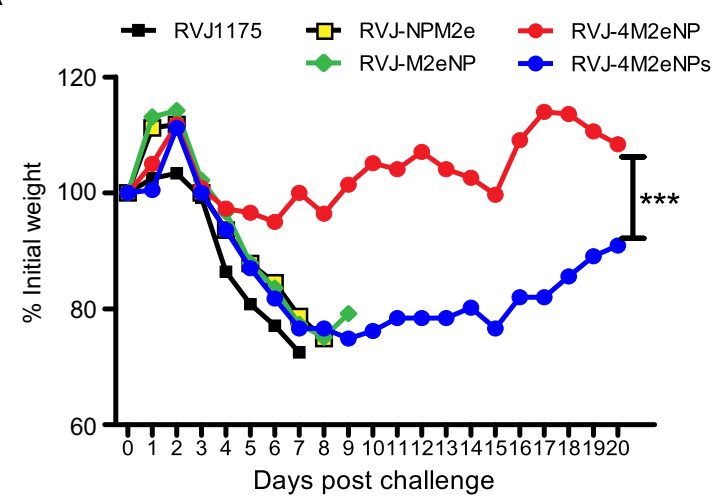

B

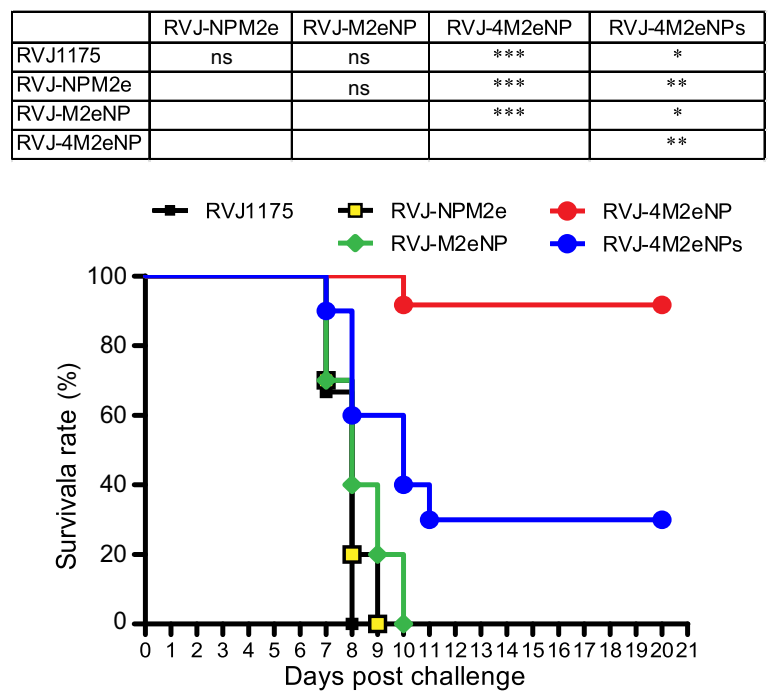

Fig. 4 Protective efficacy of recombinant vaccinia virus containing $N P$ and $M 2 e$ genes against 20 MLD50 of PR8. Five groups of mice were immunized with recombinant vaccinia viruses and challenged with 20 MLD50 of influenza virus strain PR8. Among them, mice immunized with recombinant vaccinia virus RVJ1175 served as vector control. Mice were monitored daily for 21 days after PR8 challenge. A Mice were weighed daily to monitor morbidity. Average weights in each treatment group were followed for the duration of the study, and the percentage of the original body weight was calculated based on the average starting weight for each group at day 0 . B Survival rates were calculated and compared among groups. $n s$ not significant, $* P<0.05 ; * * P<0.01 ; * * * P<0.001$.

Furthermore, we determined whether the immunogenicity of fusion antigens is influenced by the order of NP and M2e. Our data showed that RVJ-NPM2e and RVJM2eNP induced similar antigen-specific $\operatorname{IgG}$ response (Fig. 3A, RVJ-NPM2e vs RVJ-M2eNP). However, RVJM2eNP induced significantly more NP147-155- as well as M2e-specific SFCs than the former one (Fig. 3B, RVJNPM2e vs RVJ-M2eNP), suggesting that M2e at $\mathrm{N}$ terminal of the fusion antigen is a favorable configuration to induce NP- and M2e-specific cellular immunity. Moreover, our data indicated that four copies of M2e preceding NP
(4M2e-NP) is more robust toward inducing NP- and M2especific humoral immune response (Fig. 3A, RVJ-M2eNP vs RVJ-4M2eNP) and protection (Fig. 4B, RVJ-M2eNP vs RVJ-4M2eNP). In addition, we observed that only RVJ4M2eNP immunization induced significantly higher levels of IL-2 and IL-10 cytokines in mice when stimulation with the M2e pooled peptides (Fig. 3C, RVJ-M2eNP).

Significant progress is made to develop influenza vaccine using viral vectors (Kim et al. 2013; Li et al. 2013; Tutykhina et al. 2018; Dhanwani et al. 2016). Several recombinant viral vaccines have been constructed based on modified vaccinia virus Ankara expressing HA, NP, M1, and PB1 antigens (Coughlan et al. 2018; Mullin et al. 2016; Di Mario et al. 2017; Altenburg et al. 2017). Vaccinia virus Tiantan strain, developed in China as a vaccine against smallpox, has also been used as viral vector in numerous studies to develop recombinant viral vaccines. In this study, the vaccinia virus Tiantan strain was used to construct recombinant vaccinia viruses expressing fusion antigens with different configurations. The recombinant vaccinia virus expressing 4M2e and full-length NP fusion antigen induced strong cross-protection (92\%) against a lethal heterosubtypic PR8 challenge at $20 \mathrm{MLD}_{50}$ and thus regarded as the optimal one among the four constructs. In the previous study, we expressed the fusion protein NM2e in an $E$. coli system, and determined its immunogenicity in $\mathrm{BALB} / \mathrm{c}$ mice. However, it is inconvenient and time consuming to express and purify much more alternatives. Moreover, it is impossible to express target antigens in an E. coli system successfully sometimes. Compared with an $E$. coli system, the recombinant vaccinia virus system has characteristics such as high multiplication capacity, no requirement to purify proteins, and high-efficiency to determine the immunogenicity of target antigens in animal models (Coughlan et al. 2018; Mullin et al. 2016). In fact, we succeeded in constructing and determining the antigenicity of several fusion antigens of NP and M2e efficiently.

In summary, immunization with a $4 \times \mathrm{M} 2 \mathrm{e}$ and NP fusion antigen not only induced NP-specific humoral and cellular immune response, but also increased M2e-specific immunity, which together led to superior cross-protection against heterosubtypic PR8 at $20 \mathrm{MLD}_{50}$. We concluded that the cross- protection potential correlates with both NP and M2e-specific humoral and cellular immune responses induced by RVJ-4M2eNP. However, the recombinant RVJ4M2eNP containing $4 \times \mathrm{M} 2 \mathrm{e}$ and full-length NP could not induce significant neutralizing antibodies, which help to reduce the severity and duration of the illness. Therefore, it is necessary to introduce conserved B-cell epitopes of HA to induce an enhanced cross-neutralizing response and protective potency (Koday et al. 2017). Future studies should focus on the development of universal influenza 
vaccines containing multiple antigens to induce broad neutralizing responses, ADCC, and broad cellular immune responses.

Acknowledgements This work was supported by grant from the National Key Plan for Scientific Research and Development of China (2016YFC1200200). The authors gratefully acknowledge Professor Xiangmin Zhang (Wayne State University, Detroit, MI USA) for the revision of the manuscript in English.

Author Contributions RL and WW designed the experiments. WW, $\mathrm{HB}$, and WX carried out the experiments. RL and WW analyzed the data. WW and TW wrote the paper. WW, TW checked and finalized the manuscript. All authors read and approved the final manuscript.

\section{Compliance with Ethical Standards}

Conflict of interest The authors declare that they have no conflict of interest.

Animal and Human Rights Statement The whole study was approved by the Administrative Committee on Animal Welfare of the National Institute for Viral Disease Control and Prevention, Chinese Center for Disease Control and Prevention (Laboratory Animal Care and Use Committee Authorization, permit number 2016022910). All institutional and national guidelines for the care and use of laboratory animals were followed.

\section{References}

Adler-moore J, Munoz M, Kim H, Romero J, Tumpey T, Zeng H, Petro C, Ernst W, Kosina S, Jimenez G, Fujii G (2011) Characterization of the murine $\mathrm{Th} 2$ response to immunization with liposomal M2e influenza vaccine. Vaccine 29:4460-4468

Altenburg AF, Magnusson SE, Bosman F, Stertman L, De Vries R, Rimmelzwaan GF (2017) Protein- and modified vaccinia virus Ankara-based influenza virus nucleoprotein vaccines are differentially immunogenic in BALB/c mice. Clin Exp Immunol 190:19-28

Alvarez P, Zylberman V, Ghersi G, Boado L, Palacios C, Goldbaum F, Mattion N (2013) Tandem repeats of the extracellular domain of matrix 2 influenza protein exposed in Brucella lumazine synthase decameric carrier molecule induce protection in mice. Vaccine 31:806-812

Chuai X, Xie B, Chen H, Tan X, Wang W, Huang B, Deng Y, Li W, Tan W (2018) The immune response of rhesus macaques to novel vaccines comprising hepatitis B virus S, PreS1, and Core antigens. Vaccine 36:3740-3746

Coughlan L, Sridhar S, Payne R, Edmans M, Milicic A, Venkatraman N, Lugonja B, Clifton L, Qi C, Folegatti PM, Lawrie AM, Roberts R, de Graaf H, Sukhtankar P, Faust SN, Lewis DJM, Lambe T, Hill AVS, Gilbert SC (2018) Heterologous two-dose vaccination with simian adenovirus and poxvirus vectors elicits long-lasting cellular immunity to influenza virus A in healthy adults. EBioMedicine 29:146-154

Dhanwani R, Zhou Y, Huang Q, Verma V, Dileepan M, Ly H, Liang Y (2016) A novel live Pichinde virus-based vaccine vector induces enhanced humoral and cellular immunity after a booster dose. J Virol 90:2551-2560

Di Mario G, Sciaraffia E, Facchini M, Gubinelli F, Soprana E, Panigada M, Bernasconi V, Garulli B, Siccardi A, Donatelli I,
Castrucci MR (2017) Protective immunity against influenza in HLA-A2 transgenic mice by modified vaccinia virus Ankara vectored vaccines containing internal influenza proteins. Pathog Glob Health 111:76-82

Ebrahimi SM, Dabaghian M, Tebianian M, Zabeh Jazi MH (2012) In contrast to conventional inactivated influenza vaccines, $4 \times$ M2e.HSP70c fusion protein fully protected mice against lethal dose of $\mathrm{H} 1, \mathrm{H} 3$ and $\mathrm{H} 9$ influenza $\mathrm{A}$ isolates circulating in Iran. Virology 430:63-72

Emanuel EJ, Wertheimer A (2006) Who should get influenza vaccine when not all can. Science 312:854-855

Erbelding EJ, Post DJ, Stemmy EJ, Roberts PC, Augustine AD, Ferguson S, Paules CI, Graham BS, Fauci AS (2018) A universal influenza vaccine: the strategic plan for the national institute of allergy and infectious diseases. J Infect Dis 218:347-354

Fenner F, Henderson DA, Arita I, Jezek Z, Ladnyi ID (1988) Smallpox and its eradication. World Health Organization, Geneva

Fujimoto Y, Tomioka Y, Takakuwa H, Uechi GI, Yabuta T, Ozaki K, Suyama H, Yamamoto S, Morimatsu M, Mai LQ (2016) Crossprotective potential of anti-nucleoprotein human monoclonal antibodies against lethal influenza A virus infection. J Gen Virol 97:2104-2116

Guo L, Zheng M, Ding Y, Li D, Yang Z, Wang H, Chen Q, Sui Z, Fang F, Chen Z (2010) Protection against multiple influenza A virus subtypes by intranasal administration of recombinant nucleoprotein. Arch Virol 155:1765-1775

Hayward AC, Wang L, Goonetilleke N, Fragaszy EB, Bermingham A, Copas A, Dukes O, Millett ERC, Nazareth I, Nguyen-VanTam JS, Watson JM, Zambon M, Johnson AM, McMichael AJ (2015) Natural T cell-mediated protection against seasonal and pandemic influenza: results of the flu watch cohort study. Am J Respir Crit Care Med 191:1422-1431

Huang B, Wang W, Li R, Wang X, Jiang T, Qi X, Gao Y, Tan W, Ruan L (2012) Influenza A virus nucleoprotein derived from Escherichia coli or recombinant vaccinia (Tiantan) virus elicits robust cross-protection in mice. Virol J 9:322

Jegaskanda S, Co MDT, Cruz J, Subbarao K, Ennis FA, Terajima M (2017) Induction of H7N9-cross-reactive antibody-dependent cellular cytotoxicity antibodies by human seasonal influenza a viruses that are directed toward the nucleoprotein. J Infect Dis 215:818-823

Kim SH, Kim JY, Choi Y, Nguyen HH, Song MK, Chang J (2013) Mucosal vaccination with recombinant adenovirus encoding nucleoprotein provides potent protection against influenza virus infection. PLoS ONE 8:1-11

Kim YJ, Ko EJ, Kim MC, Lee YN, Kim KH, Jung YJ, Kang SM (2017) Roles of antibodies to influenza A virus hemagglutinin, neuraminidase, and M2e in conferring cross protection. Biochem Biophys Res Commun 493:393-398

Koday MT, Leonard JA, Munson P, Forero A, Koday M, Bratt DL, Fuller JT, Murnane RD, Qin S, Reinhart TA, Duus K, Messaoudi I, Hartman AL, Stefano-Cole K, Morrison J, Katze MG, Fuller DH (2017) Multigenic DNA vaccine induces protective crossreactive $\mathrm{T}$ cell responses against heterologous influenza virus in nonhuman primates. PLoS One 12:e0189780

Kui X, Guan Y, Zhou Y, Yan SS, Zhang L, Li HJ, Sun MS (2015) Progress on adenovirus-vectored universal influenza vaccines. Hum Vaccines Immunother 11:1209-1222

LaMere MW, Lam HT, Moquin A, Haynes L, Lund FE, Randall TD, Kaminski DA (2011) Contributions of antinucleoprotein IgG to heterosubtypic immunity against influenza virus. J Immunol 186:4331-4339

Lee YN, Kim MC, Lee YT, Kim YJ, Kang SM (2015) Mechanisms of cross-protection by influenza virus M2-based vaccines. Immune Netw 15:213-221 
Lei H, Peng X, Jiao H, Zhao D, Ouyang J (2015) Broadly protective immunity against divergent influenza viruses by oral co-administration of Lactococcus lactis expressing nucleoprotein adjuvanted with cholera toxin B subunit in mice. Microb Cell Fact 14:111

Li Z, Gabbard JD, Mooney AJ, Gao X, Chen Z, Place RJ, Tompkins SM, He B (2013) Single-dose vaccination of a recombinant parainfluenza virus 5 expressing NP from $\mathrm{H} 5 \mathrm{~N} 1$ virus provides broad immunity against influenza A viruses. J Virol 87:5985-5993

Ma J, Yang F, Yu H, Zhou Y, Li G, Huang M, Wen F, Tong G (2013) An M2e-based synthetic peptide vaccine for influenza A virus confers heterosubtypic protection from lethal virus challenge. Virol J 10:227

MacLeod MK, David A, Jin N, Noges L, Wang J, Kappler JW, Marrack P (2013) Influenza nucleoprotein delivered with aluminium salts protects mice from an influenza A virus that expresses an altered nucleoprotein sequence. PLoS One 8:e61775

Mullin J, Ahmed MS, Sharma R, Upile N, Beer H, Achar P, Puksuriwong S, Ferrara F, Temperton N, McNamara P, Lambe T, Gilbert SC, Zhang Q (2016) Activation of cross-reactive mucosal $\mathrm{T}$ and $\mathrm{B}$ cell responses in human nasopharynxassociated lymphoid tissue in vitro by modified vaccinia Ankara-vectored influenza vaccines. Vaccine 34:1688-1695

Muñoz-Medina JE, Sánchez-Vallejo CJ, Méndez-Tenorio A, MonroyMuñoz IE, Angeles-Martínez J, Santos Coy-Arechavaleta A, Santacruz-Tinoco CE, González-Ibarra J, Anguiano-Hernández YM, González-Bonilla CR, Ramón-Gallegos E, Díaz-Quiñonez JA (2015) In silico identification of highly conserved epitopes of influenza A H1N1, H2N2, H3N2, and H5N1 with diagnostic and vaccination potential. Biomed Res Int 2015:813047

Nahampun HN, Bosworth BT, Cunnick JE, Mogler M, Wang K (2015) Expression of H3N2 nucleoprotein in maize seeds and immunogenicity in mice. Plant Cell Rep 34:969-980

Roti M, Yang J, Berger D, Huston L, James EA, Kwok WW (2008) Healthy human subjects have CD4+ T cells directed against H5N1 influenza virus. J Immunol 180:1758-1768

Ruan L (2013) Research and application of vaccinia virus Tiantan strain vector. J Microbes Infect 8:2-8 (in Chinese)

Schotsaert M, De Filette M, Fiers W, Saelens X (2009) Universal M2 ectodomain-based influenza A vaccines: preclinical and clinical developments. Expert Rev Vaccines 8:499-508

Tutykhina I, Esmagambetov I, Bagaev A, Pichugin A, Lysenko A, Shcherbinin D, Sedova E, Logunov D, Shmarov M, Ataullakhanov R, Naroditsky B, Gintsburg A (2018) Vaccination potential of B and T epitope-enriched NP and M2 against influenza A viruses from different clades and hosts. PLoS One 13:e0191574

Wang W, Huang B, Deng Y, Wang X, Tan W, Ruan L (2007) Expression of influenza A3 virus (H3N2) M2 gene in vaccinia virus Tiantan strain. Chin J Virol 23:377

Wang W, Huang B, Jiang T, Wang X, Qi X, Gao Y, Tan W, Ruan L (2012) Robust immunity and heterologous protection against influenza in mice elicited by a novel recombinant NP-M2e fusion protein expressed in E. coli. PLoS One 7:e52488

Wang W, Huang B, Jiang T, Wang X, Qi X, Tan W, Ruan L (2014) Maximal immune response and cross protection by influenza virus nucleoprotein derived from $E$. coli using an optimized formulation. Virology 468:265-273

Wang W, Li R, Deng Y, Lu N, Chen H, Meng X, Wang W, Wang X, Yan K, Qi X, Zhang X, Xin W, Lu Z, Li X, Bian T, Gao Y, Tan W, Ruan L (2015) Protective efficacy of the conserved NP, PB1, and M1 proteins as immunogens in DNA- and vaccinia virusbased universal influenza A virus vaccines in mice. Clin Vaccine Immunol 22:618-630

Wen B, Deng Y, Chen H, Guan J, Chuai X, Ruan L, Kong W, Tan W (2013) the novel replication-defective vaccinia virus (Tiantan strain)-based hepatitis $\mathrm{C}$ virus vaccine induces robust immunity in macaques. Mol Ther 21:1787-1795

World Health Organization (WHO) (2019) Influenza (seasonal) fact sheet. https://www.who.int/en/news-room/fact-sheets/detail/influ enza-(seasonal). Accessed 1 Feb 2019

Yan K, Tan W, Wang H, Wang Y, Zhang X, Li Y, Ruan L (2009) SARS-CoV spike proteins expressed by the vaccinia virus Tiantan strain: secreted SQ protein induces robust neutralization antibody in mice. Viral Immunol 22:57-66

Zebedee SL, Lamb RA (1988) Influenza A virus M2 protein: monoclonal antibody restriction of virus growth and detection of M2 in virions. J Virol 62:2762-2772

Zhang X, Liu M, Liu C, Du J, Shi W, Sun E, Li H, Li J, Zhang Y (2011) Vaccination with different M2e epitope densities confers partial protection against $\mathrm{H} 5 \mathrm{~N} 1$ influenza A virus challenge in chickens. Intervirology 54:290-299

Zhang N, Zheng B-J, Lu L, Zhou Y, Jiang S, Du L (2015) Advancements in the development of subunit influenza vaccines. Microbes Infect 17:123-134

Zhao G, Du L, Xiao W, Sun S, Lin Y, Chen M, Kou Z, He Y, Lustigman S, Jiang S, Zheng B-J, Zhou Y (2010a) Induction of protection against divergent $\mathrm{H} 5 \mathrm{~N} 1$ influenza viruses using a recombinant fusion protein linking influenza M2e to Onchocerca volvulus activation associated protein-1 (ASP-1) adjuvant. Vaccine 28:7233-7240

Zhao G, Sun S, Du L, Xiao W, Ru Z, Kou Z, Guo Y, Yu H, Jiang S, Lone Y (2010b) An H5N1 M2e-based multiple antigenic peptide vaccine confers heterosubtypic protection from lethal infection with pandemic $2009 \mathrm{H} 1 \mathrm{N1}$ virus. Virol J 7:151

Zheng M, Liu F, Shen Y, Wang S, Xu W, Fang F, Sun B, Xie Z, Chen Z (2015) Cross-protection against influenza virus infection by intranasal administration of nucleoprotein-based vaccine with compound 48/80 adjuvant. Hum Vaccines Immunother 11:397-406

Zhou C, Zhou L, Chen Y (2012) Immunization with high epitope density of M2e derived from 2009 pandemic H1N1 elicits protective immunity in mice. Vaccine 30:3463-3469 